\title{
Harvesting can stabilize population fluctuations and buffer the impacts of climate change
} \author{
Brage Hansen ${ }^{1}$ \\ ${ }^{1}$ Norwegian University of Science and Technology \\ ${ }^{2}$ Centre for Biodiversity Dynamics \\ ${ }^{3}$ Norwegian Institute for Nature Research \\ ${ }^{4}$ Norges teknisk-naturvitenskapelige universitet \\ ${ }^{5}$ University of Guelph \\ ${ }^{6}$ The James Hutton Institute \\ ${ }^{7}$ Norwegian University of Life Sciences
}

Bart Peeters $^{1}$, Vidar GrØtan ${ }^{1}$, Marlène Gamelon ${ }^{2}$, Vebjørn Veiberg ${ }^{3}$, Aline Magdalena Lee $^{4}$, John Fryxell ${ }^{5}$, Steve Albon ${ }^{6}$, Bernt-Erik Sæther ${ }^{2}$, Steinar Engen ${ }^{4}$, Leif Loe ${ }^{7}$, and

February 12, 2021

\begin{abstract}
Harvesting can magnify the destabilizing effects of environmental perturbations on population dynamics and, thereby, increase extinction risk. However, population-dynamic theory predicts that impacts of harvesting depend on the type and strength of density-dependent regulation. Here, we used population models for a range of life histories and an empirical reindeer case study to show that harvesting can actually buffer populations against environmental perturbations. This occurs because of density-dependent environmental stochasticity, where negative environmental impacts on vital rates are amplified at high population density due to intra-specific resource competition. Simulations from our population models show that even low levels of proportional harvesting may prevent overabundance, thereby dampening population fluctuations and reducing the risk of population collapse and quasi-extinction induced by environmental perturbations. Thus, depending on the species' life history and the strength of density-dependent environmental drivers, harvesting can improve population resistance to increased climate variability and extreme weather expected under global warming.
\end{abstract}

\section{Hosted file}

ELEpeetersMS_ClimateHarvest.pdf available at https://authorea.com/users/395263/articles/ 508565-harvesting-can-stabilize-population-fluctuations-and-buffer-the-impacts-ofclimate-change 